$»$ Blut ist dicker als Wasser.« - Wozu verpflichtet Familie?

Bleisch, Barbara

DOI: https://doi.org/10.14361/9783839437483-003

Posted at the Zurich Open Repository and Archive, University of Zurich ZORA URL: https://doi.org/10.5167/uzh-188118

Book Section

Published Version

Originally published at:

Bleisch, Barbara (2017). »Blut ist dicker als Wasser.« - Wozu verpflichtet Familie? In: Lindenau, Mathias; Meier Kressig, Marcel. Alles relativ? : ethische Orientierungen zwischen Beliebigkeit und Verantwortung. Bielefeld: de Gruyter, 49-74.

DOI: https://doi.org/10.14361/9783839437483-003 


\title{
»Blut ist dicker als Wasser.« - Wozu verpflichtet Familie?
}

\author{
BARBARA BLEISCH
}

Der Relativismus ist, von seinem alltagssprachlichen Gebrauch her gesehen, ein zweischneidiges Schwert. Auf der einen Seite ist er negativ konnotiert, insofern er die Idee von allgemeingültigen Normen und Werten unterhöhlt und auf diese Weise den Kitt porös macht, der uns in Form universell geteilter Überzeugungen verbindet. Auf der anderen Seite beurteilen wir eine andere Idee des Relativismus als positiv: Etwas zu relativieren heißt auch, Ereignisse mit der gebotenen Leichtigkeit zu nehmen, indem man vermeintlich Dramatisches ins Verhältnis setzt zu wirklich Problematischem. »Alles ist relativ«, meint entsprechend nicht nur, dass wir universelle Wertsetzungen kritisch einstufen, sondern auch, dass wir es in der Hand haben, wie wir gewichten, und dass das Leid, das einem widerfährt, sich schrumpfen lässt, indem man den eigenen Horizont erweitert und die Relationen beachtet.

Im Kontext der Familienbeziehungen sind beide alltagssprachlichen Konzepte des Relativismus thematisch. Auf der einen Seite lässt sich Familie im zweiten Sinn gerade nicht relativieren, und dieser Umstand führt $\mathrm{zu}$ einer spezifischen Akzentuierung der Familienbeziehung: Einer Mutter zu sagen, sie solle sich über den Kontaktabbruch ihres Sohnes oder über den Tod ihrer Tochter nicht grämen, scheint unangemessen vor dem Hintergrund, dass Familienbeziehungen un- 
ersetzbar und unaufkündbar und damit von einer spezifischen Bedeutung sind. Auf der anderen Seite wird just mit Blick auf die Familienbeziehung immer öfter ein Relativismus beklagt, der an das erste Konzept des Begriffs anknüpft: Familie sei, so wird zuweilen behauptet, relativ geworden, weil ihr nicht mehr jener objektive Wert zugestanden werde, den sie eigentlich verdiene. In diesem Zusammenhang wird ein $>$ Niedergang der Familie< prophezeit, der dazu führe, dass die Familie als Stütze der Gesellschaft wegzubrechen drohe.

Die Behauptung einer Relativierung der Familie respektive deren Niedergangs ist allerdings voraussetzungsreich. Sie basiert auf einem ganz bestimmten traditionellen Familienbild, in dem Vater und Mutter und gemeinsame biologische Kinder in einem Haushalt zusammenleben, und suggeriert eine lange Geschichte der prosperierenden Dominanz dieses Modells. Tatsächlich ist die Idee der blutsverwandten Kleinfamilie aber vergleichsweise jung: Erst vor rund dreihundert Jahren avancierte sie zum normativen Modell einer schützenswerten Keimzelle der Gesellschaft und wurde in den 1950er und 1960er zum staatlich geschützten Idealbild der Familie erhoben, gepaart mit dem gesellschaftlich verbindlichen Lebensplan »Love, marriage, baby carriage $\ll .{ }^{1}$ Doch bereits die 1968er Bewegung kritisierte dieses Familienbild als einen Ort der Unterdrückung insbesondere der Frau, die auf eine autonome Lebensgestaltung zugunsten des Familienglücks zu verzichten hatte, während der Mann meist eine berufliche Karriere verfolgen konnte. Der aufziehende gesellschaftliche Wandel wurde befeuert durch die technischen Fortschritte in der Reproduktionsmedizin und der wachsenden Gleichberechtigung, die auch zu einem Anstieg der Scheidungsraten und entsprechend zu mehr Stief- und Patchworkfamilien führte. Damit wurde die Familie aber gerade nicht relativiert im zweiten Sinne des Relativismus, sondern vielmehr diversifiziert, also durch neue Familienformen erweitert und letztlich sogar gestärkt (vgl. Bernhard 2014: 472ff.). Denn Familien werden heute sehr bewusst ge-

1 In der Bundesrepublik Deutschland wurde sie im Grundgesetz (Art. 6) verankert. 
plant, und Kinder genießen in unseren westlichen Gesellschaften sogar den Status von Prestigeobjekten eines geglückten Lebens. Außerdem trifft eine Relativierung der Familie auch deshalb nicht zu, weil die allermeisten Personen nach wie vor ganz eindeutig zu sagen vermögen, wer zu ihrer Familie gehört und wen sie als ihre Mutter und Vater betrachten. Dies gilt selbst vor dem Hintergrund, dass viele Kinder mehr Elternteile im Sinne von Erziehungsverantwortlichen haben als genetische Erzeuger. Das ist an sich nicht einmal neu: Das Sprichwort »Blut ist dicker als Wasser « hat sich schon vor Jahrhunderten nicht allein auf Biologie, sondern auch auf die Zugehörigkeit zur Sippe bezogen, der man entstammt. Familienbande sind nicht einfach genetische Abstammungsverhältnisse, sondern robuste Geflechte, in die die eigene Biographie eingewoben ist mitsamt unserer Identität, unseren Gewohnheiten, unseren Perspektiven auf die Welt, die wir als Erwachsene zum Ausgangspunkt nehmen, um neue Weltansichten hinzuzugewinnen. Die Geflechte haben dabei nicht nur eine unvergleichliche Prägekraft für unsere Identität; wir bleiben auch zeitlebens in ihren Fäden verwoben. Wie entfernt wir voneinander leben mögen, wie irritierend wir die gegenseitigen Ansichten finden können, ja, wie sehr wir uns sogar verkrachen können - »bis dass der Tod uns scheidet« bleiben wir alle Sohn oder Tochter, vielleicht Vater oder Mutter, in vielen Fällen Schwester oder Bruder. Die Familienbande ist deshalb Schicksal und zwar über die Blutsbande hinaus, und sie hat, insofern sie keine Deutungsoffenheit zulässt, stets einen »Beigeschmack von Wahrheit«, wie der österreichische Dichter Karl Kraus einmal gesagt haben soll.

Dennoch brechen die Klagen über einen >Niedergang der Familie< nicht ab. Es scheint also jenen, die in diese Klage einstimmen, nicht darum zu gehen, dass sie befürchten, es gebe demnächst keine Familien mehr (vgl. Archard 2015; Gheaus 2015). Vielmehr ist sie Ausdruck der Sorge, der familiäre Zusammenhalt im Sinne einer geteilten Verantwortung, einer verlässlichen gegenseitigen Fürsorge bröckle. Damit wird eine Verbindung angemahnt zwischen Relativierung und Verantwortungsabnahme, die auch im Ausschreibungstext der Vadian Lectures herausgestrichen wird: Weil heute Vieles relativ scheint, 
nimmt - so wird zumindest unterstellt - auch die Verantwortung füreinander ab. So gewendet, ist Familie kein soziologisches Konstrukt eines Mehrgenerationengefüges, sondern ein normatives Ideal, in dem Menschen verlässlich füreinander da sind.

Nun ist es freilich eine empirische Frage, ob Familienmitglieder heute tatsächlich weniger als früher bereit sind, sich umeinander zu kümmern. Aus philosophischer Perspektive ist zu dieser empirischen Frage nichts beizusteuern. Vielmehr setzt dieser Beitrag eine Stufe tiefer an und analysiert sozusagen die Grundlage dieser Klage. Denn bevor wir den Vorwurf erheben, Andere kämen ihrer familiären Verantwortung nicht nach, gilt es zu prüfen, worin denn ihre Verantwortung tatsächlich bestünde - oder anders gefragt: Ob Familie verpflichtet und wenn ja, wozu?

Diese Frage mag vielleicht trivial erscheinen: Was, wenn nicht Familie, verpflichtet uns? Tatsächlich scheinen wir in unserer alltäglichen Lebenspraxis dauernd mit der Existenz familiärer Pflichten zu rechnen. Die Entschuldigung, wegen >familiärer Pflichten< verhindert gewesen zu sein, akzeptieren wir in den meisten Kontexten ohne Zögern. Ob ein Elternteil im Sterben liegt, die Schwester schwer erkrankt ist oder gerade ein neues Enkelkind geboren wurde - familiäre Beziehungen geben uns offenbar eine Vielzahl von Gründen, die uns auch gegenüber anderen darin legitimieren, unsere Arbeit zu unterbrechen oder Termine abzusagen (vgl. Betzler/Bleisch 2015).

Das gilt natürlich im Besonderen mit Blick auf kleine Kinder, für die die jeweiligen Eltern mit ihrer Anerkennung der Mutter- respektive der Vaterschaft Obhut-, Fürsorge- und Erziehungspflichten übernehmen, die bestehen, bis das Kind seine Volljährigkeit erreicht hat. Doch diese Elternpflichten sind hier nicht thematisch. Denn wenn wir danach fragen, ob Familie an sich verpflichte, wollen wir wissen, ob uns die familiäre Bande auch dann Aufgaben überträgt, wenn wir es nicht mehr mit der Fürsorge gegenüber abhängigen Kleinkindern zu tun haben, sondern wenn wir als Ebenbürtige, aber eben Verwandte, voreinander stehen. Im Folgenden fokussiere ich zur Bearbeitung dieser Frage auf eine familiäre Konstellation, nämlich auf die Frage, ob er- 
wachsene Kinder ihren Eltern etwas schulden. Man kann diese Frage auch salopp zuspitzen: Gibt es Rabentöchter, gibt es Rabensöhne? Kinder also, die - analog zur Rabenmutter - ihren Pflichten als Nachkommen nicht zur Genüge nachkommen und ihre Eltern ungerechtfertigt vernachlässigen (vgl. Bleisch 2015)?

Die Idee, dass erwachsene Kinder ihren Eltern etwas schulden, hat eine lange Tradition. Bereits die Bibel gebietet an prominenter Stelle, nämlich im Dekalog, Vater und Mutter zu ehren, und die meisten Menschen halten es für geboten, den eigenen Eltern zumindest mit einem größeren Maß an Interesse, Respekt und Fürsorge zu begegnen, als sie dies anderen Personen gegenüber tun - und zwar auch dann, wenn sie selber aus dem Kontakt mit den Eltern nicht mehr allzu viel schöpfen. Gleichzeitig kommt es immer wieder zu Familienabbrüchen, in denen Kinder ihren Eltern den Kontakt aufkündigen und Eltern sich in einschlägigen Internetforen ausführlich beklagen über ihre undankbaren Kinder. Es ist also durchaus strittig, was Kinder ihren Eltern schulden. In der Debatte um Familienabbrüche zeigt sich allerdings nur eine Extremform der ungleichen Bedürfnisse und gegenseitigen Ansprüche, die Eltern und ihre erwachsenen Kinder aneinander haben können. Vor dem Familienabbruch stellen sich oft harmlosere Fragen wie jene, wie oft eine erwachsene Tochter ihre betagte Mutter besuchen sollte; ob der Sohn sich an der Pflege seines Vaters beteiligten müsste; ob die Kinder Weihnachten auf den Malediven verbringen dürfen, wenn die Eltern das obligate Familienfest anberaumt haben; und ob Großeltern das Recht haben, ihre Enkelkinder zu sehen, wenn der Kontakt zu den eigenen Kindern nicht der beste ist. Alle diese Problemstellungen fundiert die eine große Frage, wozu >Kindschaft< verpflichtet - der Umstand also, dass wir alle Söhne oder Töchter von Eltern sind. Was schulden erwachsene Kinder ihren Eltern? Und wie lässt sich eine solche Pflicht allenfalls begründen? Dieser Frage gehe ich im Folgenden nach.

Ich werde zuerst zwei Vorschläge diskutieren, wie die filiale Verantwortung (also die Verantwortung erwachsener Kinder gegenüber ihren Eltern) bereits begründet worden ist, nämlich mit dem Hinweis 
auf Schuld und Dankbarkeit. Beide Vorschläge sind jedoch mit erheblichen Schwierigkeiten konfrontiert, die unter anderem darauf zurückzuführen sind, dass die Frage der Verpflichtung vor allem mit Blick auf die sogenannte Überalterung unserer Gesellschaft und der damit verbundenen steigenden Pflegekosten gestellt worden ist. Dieser Blick verengt jedoch das Nachdenken über die erwachsene Elternkindbeziehung in problematischer Weise. Ich schlage deshalb im zweiten Abschnitt einen Perspektivenwechsel vor weg von einem transaktionalen Modell der familiären Verantwortung hin zu einem relationalen Modell. In diesem Kontext wird das Konzept der relationalen Verletzlichkeit relevant. Im dritten Abschnitt diskutiere ich, was Kinder ihren Eltern schulden vor dem Hintergrund dieser spezifischen Verletzlichkeit. Ich komme zum Schluss, dass Kinder ihren Eltern nichts schulden, dass sie aber sowohl mit Blick auf das eigene Wohl, wie auf das Wohl ihrer Eltern guten Grund haben, dieser spezifischen Verletzlichkeit in ihrem Handeln Rechnung zu tragen und sich dieser stets bewusst zu sein.

\section{SCHULD UND DANKBARKEIT}

Als Begründung für eine spezielle Verpflichtung von Kindern gegenüber ihren Eltern wird oft darauf verwiesen, was Eltern für ihre Kinder in deren Kindheit getan haben. Dieses >Schuldnermodell< filialer Pflichten hat eine lange philosophische Tradition. So war schon Aristoteles der Meinung, Kinder hätten ihren Eltern gegenüber spezifische Pflichten zur Rückzahlung einer Schuld aufgrund der vielen Mühen und Aufwendungen, welche die Erziehungsarbeit für die Eltern mit sich gebracht hat (vgl. Aristoteles 1995). Die Idee wäre demzufolge, dass Eltern und ihre Kinder so etwas sind wie Gläubiger und Schuldner: Eltern haben ihren Kindern durch ihre Erziehungsarbeit ein Darlehen gegeben, das Kinder im Erwachsenenalter abzutragen haben in Form von Zuwendung den eigenen Eltern gegenüber. 
Diese Analogie ist allerdings aus verschiedenen Gründen wenig überzeugend. Erstens konfrontiert uns dieses $>$ Schuldnermodell< mit einem epistemischen Problem: Worin genau bestünde die entsprechende Leistung für das, was Eltern ihren unmündigen Kindern gegeben haben? Es scheint sehr schwierig, wenn nicht unmöglich, diesbezüglich Angaben zu machen und eine vergleichbare Leistung von den erwachsenen Kindern zu fordern. Anders als im Fall der GläubigerSchuldner-Beziehung fehlt im Fall des Eltern-Kind-Verhältnisses nämlich schlicht ein Vergleichsmaßstab, der notwendig scheint, um den Inhalt der kindlichen Pflichten bestimmen zu können. Auf der Suche nach einem solchen Vergleichsmaßstab wird zweitens deutlich, dass die Schuldner-Gläubiger-Analogie in wesentlicher Hinsicht in die Irre führt: Sobald der Schuldner die bestimmbare reziproke Leistung erbringt, also z.B. das Geld zurückzahlt, sind er und sein Gläubiger >quitt<. Einen solchen Zustand des Ausgleichs herzustellen, der das Ende der Verpflichtung markiert, ist geradezu der Witz von Pflichten im Sinne des Schuldnermodells. Die Pflichten, die Kinder gegenüber ihren Eltern haben, lassen sich in diesem Bild jedoch gerade nicht einfangen. Eltern werden ihren Kindern nicht vorrechnen, dass sie sie noch drei Mal besuchen müssen, bis die Schuld abgetragen ist, die sich das Kind aufgeladen hat. Ein solcher Blick wäre unangemessen und würde eher das Ende der Beziehung markieren als die Idee stützen, dass die Beziehung weiter bestehen soll. Doch selbst wenn man diese Unklarheiten außer Acht lässt, stellt sich ein drittes Problem: Im Fall des Lebensgeschenks und auch der Sorge um das Kleinkind fehlt schlicht die >Vereinbarung<, die Schuldnermodellen zugrunde liegt. Kinder haben um ihr Leben ebenso wenig wie um ihre Erziehung und Pflege gebeten, und es scheint problematisch, jemanden in die Pflicht zu nehmen, weil man ihm etwas gegeben hat, wovon man gar nicht wusste, ob er oder sie es haben wollte. Aus diesem Grund gibt es auch keine Pflicht, für die empfangenen Gaben eine Gegenleistung zu erbringen.

Allgemeiner formuliert bringt das Schuldnermodell die Idee der fairen Kostenverteilung ins Spiel: Nicht nur die Geburt, sondern das 
Aufziehen von Kindern ist mit Lasten verbunden, die - so der Gedanke - unter denen, die den Profit haben, fair verteilt werden sollten. Eltern sind diesbezüglich gleichsam in Vorleistung gegangen, und die kindlichen Pflichten ihnen gegenüber dienen dem Ausgleich der so entstandenen Schieflage. Doch warum sollte das bloße Faktum des Profits jemanden zur Beteiligung an den Kosten verpflichten? Ich profitiere beispielsweise enorm davon, dass die über mir wohnende Nachbarin eine leidenschaftliche Cineastin ist, und jeden zweiten Abend im Kino verbringt, denn das erlaubt mir, in ihrer Abwesenheit laut Musik zu hören. Gleichwohl wäre es absurd, wenn sie allein aufgrund dieser Profite von mir verlangen würde, mich an den Kosten für ihre Kinokarten zu beteiligen. Das hängt nicht zuletzt damit zusammen, dass sie mit den Kinobesuchen zunächst einmal sich selber einen Gefallen tut, und dies gilt ganz ähnlich auch für die Elternkindbeziehung: Mit der Zeugung von Kindern haben Eltern zuallererst etwas für sich getan, nämlich ihrem Wunsch entsprochen, Nachwuchs in die Welt zu setzen. Und weil dem so ist, leuchtet es keinesfalls ohne Weiteres ein, dass ihre Kinder ihnen dafür eine Gegenleistung schulden. Das Schuldnermodell scheint daher nicht geeignet um zu begründen, weshalb ein erwachsenes Kind seinen Eltern verpflichtet sein sollte.

Das klingt hart und, wie man vielleicht einwenden könnte, reichlich undankbar. Doch mit der Berufung auf die Undankbarkeit des Kindes wird die Argumentationsebene gewechselt von der These einer Schuldhaftigkeit zur Behauptung einer Pflicht der Dankbarkeit. Vielleicht sollten Kinder ihren Eltern ja wirklich dankbar sein für das, was diese für sie getan haben? Was liegt näher als der Gedanke, dass wir der Frau, die uns unter Schmerzen geboren hat, in besonderer Weise dankbar sein sollten und deshalb verpflichtet sind? Dass wir dem Vater, der uns über Jahre hinweg begleitete und auf uns im besten Fall auch Acht gab, Dank schulden? Dankbarkeit für eine glückliche Kindheit oder für fröhliche Stunden, die man im Kreis der Familie zugebracht hat, ist zumindest eine weit verbreitete Haltung, die uns alles andere als unangemessen scheint. Doch folgt aus dieser offenbar geteilten Praxis eine Pflicht? Ich meine nein. Eine Haltung der Dankbar- 
keit kann man vermissen; sie einzufordern ist hingegen unmöglich, denn Haltungen lassen sich nicht gebieten, sondern lediglich kultivieren. Und selbst wenn es möglich wäre, eine entsprechende Haltung einzufordern, sich also zeigen würde, dass wir dankbar sein sollten für die Familienkonstellation, von der wir profitieren durften, ergeben sich daraus keine konkreten Forderungen, die Eltern an ihre Kinder stellen könnten. Verlieren wir beispielsweise unseren Geldbeutel und ein ehrlicher Finder schickt uns diesen zurück, sollten wir diesem möglicherweise wirklich dankbar sein. Wir schreiben in einer solchen Situation eine Karte, schicken Blumen, zeigen uns erkenntlich. Abgesehen von einer Geste, die der Dankbarkeit Ausdruck verleiht, bestehen aber keine weiteren Pflichten. Zugegeben ist das, was Kinder von ihren Eltern erhalten, von einer ganz anderen Dimension als ein verlorener Geldbeutel. Aber selbst wenn wir dies anerkennen, fragt sich, was daraus genau an Dankbarkeitsgesten folgen sollte. Eine junge Frau kann mit guten Gründen zu ihren Eltern sagen: »Ich bin Euch ewig dankbar, für alles, was ihr mir geschenkt habt, aber jetzt möchte ich meine eigenen Wege gehen.«Was berechtigt die Eltern zum Urteil, ihr Weggang sei Ausdruck von Undankbarkeit? Dankbarkeit steht uns als Haltung in vielen Fällen gut an, doch aus ihr folgen keine berechtigten Ansprüche auf konkrete Güter oder Verhaltensweisen des Gegenübers.

Halten wir an dieser Stelle kurz inne: Die bisherigen Versuche zu zeigen, weshalb Kinder ihren Eltern speziell verpflichtet sein könnten, fokussierten auf die Frage, was Eltern ihren Kindern in deren Kindheit gegeben haben und was sie sozusagen als Gegenleistung von ihrem Nachwuchs erwarten können. Der Grund für diese Engführung der Debatte findet sich unter anderem im Umstand, dass mit der zunehmenden Überalterung unserer Gesellschaft und der Vereinsamung vieler betagter Menschen die Frage aufkommt, wer diesbezüglich in die Pflicht zu nehmen ist, d.h. wer für die entsprechenden Hilfeleistungen finanziell aufzukommen hat. ${ }^{2}$

2 Vgl. dazu bspw. das sogenannte >Rabenvater-Urteil<, in dem der deutsche Bundesgerichtshof einen Beamten verpflichtete, sich mit rund 9.000 Euro 
In diesem Zusammenhang liegt es offenbar nahe, darauf zu verweisen, dass Kinder von ihren Eltern vorgängig massiv profitiert haben und dass sie entsprechend etwas zurückgeben müssen. Dies ist die Grundidee der Schuldner- wie der Dankbarkeitsmodelle. Sie stehen im Kern für das, was ich eine Moral der Transaktion nenne: Moralisch zu handeln heißt in den entsprechenden Kontexten, einen Ausgleich herzustellen für Leistungen, die einseitig erbracht worden sind. Dieses Moralverständnis fundiert viele soziale Beziehungen. Doch einerseits beruht es auf einem Bild von autonomen Beziehungspartnern, die frei entscheiden, welche Güter sie tauschen wollen und darüber allenfalls sogar eine Vereinbarung treffen. Dieses Bild passt aber gerade nicht zur Familie, in der die Beteiligten sich nicht frei wählen, sondern einander nolens volens gegeben sind. Andrerseits führt es die Frage, was wir einander schulden, zu Unrecht auf den Austausch von Gütern und Fürsorge eng. Doch die fraglichen Pflichten erschöpfen sich nicht in Hilfsgeboten, denn viele Fragen, die uns im Zusammenhang mit unseren Eltern umtreiben, lassen sich nur adäquat erfassen, wenn wir von der Debatte um die Finanzierung der Fürsorge und der Pflege Abstand nehmen. So fragen sich erwachsene Kinder beispielsweise auch, ob sie heimlich heiraten oder ihren Eltern die Enkel vorenthalten dürfen oder ob sie das Alkoholproblem des Vaters ansprechen müssten. Es geht also bei den fraglichen Pflichten keinesfalls ausschließlich oder vorrangig um Hilfsgebote, sondern ebenso um Diskretionspflichten und Interventionsgebote. Um die Frage nach der Begründung der entsprechenden Pflichten in ihrer Gesamtheit beantworten zu können, müssen

an den Kosten für das Pflegeheim zu beteiligen, in dem dessen Vater bis zu seinem Tod lebte, obwohl der Vater den Kontakt zu seinem Sohn abgebrochen hat, als er achtzehn Jahre alt war, und ihn testamentarisch enterbte. Der Bundesgerichtshof entschied in letzter Instanz, der Sohn müsse seiner Unterhaltspflicht nachkommen. Auch wenn der Vater das familiäre Band zerschnitten habe, habe er sich in den ersten achtzehn Lebensjahren seines Sohnes um ihn gekümmert und damit seine Elternpflichten im Wesentlichen erfüllt (Beschluss des Bundesgerichtshof BGH XII ZB 607/12). 
wir aber vor allem eines tun, nämlich die Elternkindbeziehung besser verstehen und danach fragen, wozu die Beziehung selbst - unabhängig von Vorleistungen - verpflichtet. Ich plädiere also im Kontext der Familienbeziehungen für einen Perspektivenwechsel: weg vom transaktionalen Modell der Moral hin zu einem relationalen Modell der Moral, das auf der Beziehung, nicht auf dem Austausch von Gütern beruht.

\section{Relationale Moral und Verletzlichkeit}

Dabei stellt sich natürlich sogleich die Frage, ob Beziehungen an sich verpflichten, ohne auf den Austausch von Gütern Bezug zu nehmen?

Betrachten wir dazu zuerst eine mögliche Form der persönlichen Beziehung, die Freundschaft. Tatsächlich sind viele der Meinung, Freund oder Freundin zu sein, heiße gerade, der befreundeten Person gegenüber speziell verpflichtet zu sein. Dies gilt nicht nur für die gern zitierten Nothilfeszenarien (Freunde sind demnach als Erste aus dem Teich oder dem brennenden Haus zu retten), sondern auch mit Blick auf andere Gebote, etwa Diskretionspflichten (was einem eine Freundin anvertraut, hat man für sich zu behalten) oder Interventionsgebote (gute Freunde sollten einander vor existentiellen Fehlern bewahren). Dabei steht gerade nicht die Fairness einer Transaktion im Vordergrund, also die Frage, ob einem Freund jenes Maß an Zuneigung geschenkt wird, das er einem selber hat angedeihen lassen. Derartige >Aufrechnungen< sind eher ein Zeichen dafür, dass es mit der Beziehung nicht zum Besten steht, als dass man sich einander zugeneigt fühlt. Freundschaftspflichten sind nicht von der Art, dass sie irgendwann gänzlich erfüllt oder >abgehakt< wären, wie dies bei transaktionalen Pflichten wie Schuldner- oder Dankespflichten der Fall ist. Vielmehr ist die Erfüllung entsprechender Pflichten für die Beziehung konstitutiv: Wenn eine Freundin nicht mehr bereit ist, ihren Freund zu unterstützen, die Geheimnisse, die er ihr anvertraut für sich zu behalten, und im Streitfall mit Dritten für ihn Partei zu ergreifen, ist dies ein 
Zeichen dafür, dass sie womöglich nicht mehr an der Freundschaft interessiert ist.

Nun mag diese Position zwar lebensweltlich überzeugend sein, philosophisch hat sie jedoch einige Haken. Denn die These, dass Pflichterfüllung konstitutiv ist für eine Beziehung, die ihrerseits neue Pflichten generiert, ist einerseits zirkulär. Andrerseits mutet es mit der Idee von moralischen Pflichten nur schwer vereinbar an, dass man seinen Verpflichtungen dadurch entgehen kann, dass man selbständig die normativen Voraussetzungen der Situation ändert - also im vorliegenden Fall die Freundschaftsbeziehung aufkündigt. Allerdings entspricht diese Darstellung der fluiden Freundschaft auch gar nicht dem Ideal des >guten Freundes $<$, der einem treu verbunden bleibt, egal was kommen mag. Das betonte schon Aristoteles in seiner Nikomachischen Ethik und wiederholte im 18. Jahrhundert Michel de Montaigne (1998) in seinem Essay Von der Freundschaft. Wer sich aus einer Freundschaft verabschiedet, einfach weil er oder sie das Interesse am gewohnten Gegenüber verloren hat, scheint nicht verstanden zu haben, worum es in einer Freundschaft wirklich geht. Dies gesagt, kann es natürlich dennoch zu Zerwürfnissen zwischen Freunden kommen oder zu einem Gefühl der Verflachung der Beziehung, die vielleicht dazu führt, dass man sich aus den Augen verliert. Momente der Reibung und vielleicht auch der Trennung scheinen mir nun aber besonders informativ zu sein für das Nachdenken über Freundschaftspflichten. Der Vorwurf, beispielsweise ungerechtfertigt verlassen oder verraten worden $\mathrm{zu}$ sein, bringt Erwartungen zum Ausdruck, die auf eine Verletzung von Pflichten hinzudeuten scheinen. Tatsächlich kommt die Moral in Beziehungen meist erst dann ins Spiel, wenn Beziehungen brüchig werden. Beziehungen sind moralisch gesehen insofern störungsanfällig, als sie stets mit der Möglichkeit von Verrat und Verlust einhergehen. Denn indem wir lieben und vertrauen, indem wir uns auf andere Menschen einlassen und gemeinsame Projekte mit ihnen teilen, machen wir uns auch verletzlich.

Der Begriff der Verletzlichkeit hat unterschiedliche Dimensionen (vgl. Mackenzie/Stoljar 2000): Menschen sind erstens in einem ontolo- 
gischen Sinn verletzlich. Der Mensch ist nicht bloß ein autarkes, autonomes Individuum, wie ihn der Liberalismus zeichnet, sondern Menschen sind als wesentlich körperliche Entitäten verletzlich und aufeinander angewiesen: Sie können erkranken, verwundet werden, sie sterben (vgl. MacIntyre 1999; Butler 2004). Menschen sind zweitens sozial verletzlich: Sie können als Mitglieder einer Gesellschaft eine schlechte soziale Stellung haben, diskriminiert oder ausgegrenzt werden. Soziale Verletzlichkeit bringt immer eine Schlechterstellung einer Person im Hinblick auf ein bestimmtes Gut zum Ausdruck. So weisen sozioökonomisch schlechter Gestellte beispielsweise in vielen Gesellschaften eine höhere Morbiditätsrate auf. Diese beiden Konzepte der Verletzlichkeit - ontologisch und sozial - sind in der Ethik deshalb wichtig, weil sie ausweisen, dass wir den Menschen nicht nur als frei und unabhängig, sondern eben auch als vulnerabel denken müssen, wenn wir fragen, was wir einander schulden und an welchen Grundfesten sich moralisches Handeln zu orientieren hat. Aber für die Frage, was sich Personen in Familienbeziehungen schulden, ist ein dritter Begriff der Verletzlichkeit einschlägig, der relational, also beziehungsbasiert ist. Relationale Verletzlichkeit ergibt sich allein im Rahmen und in Abhängigkeit von Verbundenheit und gegenseitigem Vertrauen.

Relationale Verletzlichkeit weist dabei unterschiedliche Facetten auf, von denen ich an dieser Stelle drei unterscheiden möchte: Freundinnen sind erstens epistemisch vulnerabel. In den meisten Fällen kennen sie sich schon lange und sind deshalb vertraut mit den Vorlieben und Einstellungen der Anderen, wissen aber auch um ihre Schwächen und dunklen Seiten. Sie haben Erfolge geteilt, sind einander aber auch beigestanden in Situationen des Scheiterns. Sie haben sich Geheimnisse anvertraut und Vertraulichkeiten ausgetauscht. Eine tiefe persönliche Beziehung geht deshalb einher mit einem tieferen Verständnis für die andere Person. Dieses Verständnis füreinander verschafft Freunden einen epistemischen Vorteil gegenüber Außenstehenden, der ihnen in guten Zeiten erlaubt, einander besonders zu unterstützen, in schlechten Zeiten aber auch die Möglichkeit eröffnet, einander in einer Weise zu 
verletzen, zu betrügen oder auszunutzen, wie es Außenstehende nicht zu tun vermöchten.

Freunde nehmen überdies am Leben des Anderen teil. Vielleicht spielen sie regelmäßig Tennis zusammen, fahren jedes Jahr gemeinsam in den Urlaub oder machen einander zu Paten ihrer Kinder. Freundschaften bestehen wesentlich aus Ritualen, an die sich beide Seiten mehr oder minder verlässlich halten. Gibt eine Seite das Ritual auf oder erlischt einseitig das Interesse für eine geteilte Praxis, provoziert dies Irritationen, die nach einer Erklärung oder sogar Rechtfertigung rufen. Weil Freundschaft auch darin besteht, dass Erwartungen verlässlich erfüllt werden, sind Freunde zweitens erwartungsbasiert verletzlich.

Diese erwartungsbasierte Verletzlichkeit ist drittens eng verwoben mit dem Umstand, dass wir in Beziehungen auch emotional vulnerabel sind. Liebe geht einher mit emotionaler Bindung, und dieser Bindung wird ganz wesentlich Ausdruck verliehen durch geteilte Rituale, Interessen und Gewohnheiten. (Liebespaare, die sich trennen, geben entsprechend oft als Grund für die Trennung an, sie hätten nichts mehr geteilt.) Jemanden zu lieben, heißt deshalb immer auch, ein emotionales Risiko einzugehen: das Risiko verlassen, betrogen oder zurückgewiesen zu werden.

Es gibt Personen, die dieses Risiko nicht eingehen möchten und deshalb nahe Beziehungen weitgehend vermeiden. Anders als bezüglich der ontologischen und sozialen Verletzlichkeit sind wir mit Blick auf die relationale Verletzlichkeit bis zu einem gewissen Grad frei zu entscheiden, wie nahe wir andere Menschen an uns heranlassen und wie sehr wir damit das Risiko eingehen, verletzt zu werden.

Aber ganz abgesehen davon, ob es eine erfüllende Lebensform sein kann, sich aus Angst vor Verletzung gegen Beziehungen zu verwahren, haben wir in Familienbeziehungen diese Freiheit nicht: Wir hatten nie die Möglichkeit, uns frei zu entscheiden, ob wir diese Eltern haben wollen. Ebenso wenig waren die Eltern frei im Entscheid, mit dem spezifischen Kind, das das ihre ist, verbunden zu sein. Sie konnten lediglich entscheiden, ob sie überhaupt Kinder haben wollen. Aber von 
dem Moment an, in dem sie die Elternschaft anerkannten, begann auch die Beziehung zum Kind. Freilich ist auch familiäre Liebe nicht umsonst zu haben, sondern es muss in die Beziehung investiert werden. Gerade deshalb scheint ja die Idee, dass Kinder ihren Eltern etwas schulden, so naheliegend: Das elterliche Investment ist nicht nur finanziell und zeitlich, sondern auch emotional hoch. Doch der Fokus auf die Investitionen verkennt, dass die moralische Währung in persönlichen Beziehungen nicht die Schuld ist, die sich aus einem Aufrechnen mit der Vergangenheit ergibt. Es ist vielmehr die Liebe respektive deren Kehrseite: die Verletzlichkeit, die diese Beziehungen prägen.

Diese Nichtwählbarkeit der familiären Bande verschärft nun aber die relationale Verletzlichkeit in entscheidender Weise. Was deren epistemische Dimension anbelangt, haben Eltern natürlicherweise einen umfassenden Wissensvorsprung im Vergleich zu ihren Kindern, die die Kindheit ihrer Eltern nicht miterlebt haben. Die umfassende Kenntnis des eigenen Nachwuchses kann für diesen auch bedrohlich ausfallen, wenn er aus den Rollen, die ihm zugefallen sind, nicht mehr schlüpfen kann. Der Grund dafür liegt in den normalerweise recht statischen Bildern, die wir von jenen haben, die wir lieben. Da Vertrautheit auch darin besteht, die geliebten Menschen >lesen < zu können und ihre Handlungen und Haltungen bis zu einem gewissen Grad antizipieren zu können, bekunden Freunde oft Mühe damit, wenn sich diese stark verändern. Es ist eine offene Frage, wie viel Veränderung Zuneigung und Liebe ertragen kann und soll. Gerade in Familienbeziehungen zeigt sich jedoch oft, dass Entwicklungen von Personen nur schwer zugelassen werden können. Die epistemische Vulnerabilität kann sich dann zu einer Art Zwangsjacke verdichten, in der wir einander ständig vor dem Hintergrund der Familiengeschichte sehen und einordnen. Die Familiengeschichte hilft uns deshalb nicht nur in einem positiven Sinne zu verstehen, wer wir sind, wie David Velleman (2005) betont; wir können uns auch eingesperrt fühlen in der eigenen Geschichte (kritisch dazu: Haslanger 2009). Epistemische Vulnerabilität kann deshalb in Familiengefügen eine spezifische Verschärfung erfahren. 
Der zweite Aspekt dieser speziellen Akzentuierung der epistemischen Verletzlichkeit betrifft den Umstand, dass Familien stets mehrere Personen umfassen, die miteinander in einer Weise verwoben sind, wie es die Freunde einer Person normalerweise untereinander nicht sind. Wenn eine Mutter das Geheimnis ihres Sohnes ausplaudert, kann das nicht nur den Sohn verletzen, sondern auch negative Auswirkungen haben auf dessen Ehefrau, seine Schwestern, seinen Vater. Familien werden zurecht als Systeme beschrieben, und Familiengeheimnisse tabuisiert, weil sie aufzudecken das ganze System beeinträchtigen würde. Inzest ist vermutlich der problematischste Fall eines Familiengeheimnisses, das meist sehr gut gehütet wird, weil alle Vertrauenspersonen zugleich Betroffene sind - nicht nur als eigentliche Opfer, sondern auch in ihrer Rolle als Mitwisser. Zwar können solche Phänomene auch in Peer-Gruppen oder Freundeskreisen auftreten. Meist sind diese Gruppen aber doch loser, sodass es nicht zu denselben gravierenden Effekten kommt. Außerdem behaupte ich an dieser Stelle nicht, dass epistemische Verletzlichkeit in Familien kategorisch anders ausfällt als in Freundschaften. Ich sage nur, dass diese Art der Verwundbarkeit in vielen Familien eine spezielle Akzentuierung erfährt.

Dasselbe gilt auch für die erwartungsbasierte und die emotionale Vulnerabilität. Wie ich in Bezug auf Freundschaften schon gesagt habe, bestehen intime Beziehungen meist aus geteilten Praktiken und Ritualen, und der Wert der Beziehung hängt unter anderem davon ab, dass wir uns verstanden und aufgehoben fühlen, gerade weil die Verbindung in all den kleinen Vertrautheiten Bestätigung erfährt. Wir fühlen uns einander zugehörig und verbunden - und entsprechend sind wir auch verletzlich mit Blick auf das Ende dieser Zugehörigkeit und Verbundenheit. Im Vergleich zur Freundschaft erfährt aber auch dieser Typ der Verletzlichkeit in der Familie zwei Akzentuierungen:

Die erste Akzentuierung betrifft den Umstand, dass die Familienbeziehung als solche nicht beendet werden kann. Natürlich können auch Kinder den Kontakt zu ihren Eltern abbrechen und Eltern ihre Kinder verstoßen. Dennoch bleibt das Kind bis zu seinem Tod das Kind dieser spezifischen Eltern, und Eltern bleiben bis zum Tod ihres 
Kindes dessen Vater oder Mutter. Es gibt Exfreunde, Exfrauen, Expartner - aber es gibt keinen Exvater, keine Exschwester oder Extochter. Dagegen kann man durchaus Freundschaften beenden oder sich so verhalten, dass die Freundschaft leidet und früher oder später zu einem Ende kommt. Die Familienbeziehung dagegen lässt sich nicht aufkündigen. Die amerikanische Philosophin Claudia Mills (2015) sieht in dieser Nichtwählbarkeit einen speziellen Wert, weil sie meist damit einhergehe, dass wir bedingungslos geliebt werden: Gerade weil wir uns in unseren nahen Familienbeziehungen gegenseitig nicht wählen können, können wir auch keine Bedingungen aneinander stellen. Wir sind einander >gegeben $<$, so Mills, und müssen uns deshalb miteinander arrangieren. Dies kann zwar im Einzelfall auch nachteilig sein, doch in erster Linie ist es ein hohes Gut, über einen Vorrat an Liebe zu verfügen, den wir uns nicht erst verdienen müssen und den wir auch nicht beim kleinsten Fehltritt verlieren. Doch Mills schildert damit nur die glänzende Seite der Medaille, die unter anderem ein Grund dafür ist, weshalb Familienbeziehungen sicherlich nie leichtsinnig aufgegeben werden. Die dunkle Seite des Umstands, dass wir unsere Blutsbande nie loswerden, besteht aber darin, dass uns dies auch in einer spezifischen Weise verletzlich macht, weil man sozusagen nicht übereinander hinwegkommen kann.

Die zweite Akzentuierung betrifft den Umstand, dass die Elternkindbeziehung unersetzbar ist. Wenn ein Sohn den Kontakt zu seinem Vater abbricht, kann der Vater nicht einen neuen Sohn finden. Zwar gilt dies bis zu einem gewissen Grad für alle tiefen persönlichen Beziehungen. Liebespartner hoffen zurecht, dass sie einander nicht als ersetzbar sehen, und auch langjährige gute Freunde teilen vermutlich das Gefühl, in einer Weise verstanden zu werden, wie sie nie wieder von einer anderen Person verstanden werden. Dennoch ist es selbstverständlich angemessen, eine verlassene Freundin zu trösten mit den Worten: »Gräme Dich nicht, er war sowieso nicht der Richtige für Dich, und früher oder später wirst Du Dich wieder verlieben und jemanden finden, der besser zu Dir passt. «Es wäre aber vollkommen inadäquat, Ähnliches zu Eltern zu sagen, die von ihren Kindern verlassen 
wurden, oder zu Kindern, deren Eltern nichts mehr von ihnen wissen wollen. Ist man erst einmal erwachsen, kann man nicht noch einmal neu anfangen mit der Familienbeziehung. Deshalb verschärft sich auch die emotionale Verletzlichkeit im Rahmen der Familienbande.

Zugegeben lässt sich an dieser Stelle einwenden, dass die Akzentuierungen, die ich eingeführt habe, nur formale Eigenschaften der Familienbande sind: Es ist ja nicht die Beziehung, die nicht gekündigt oder ersetzt werden kann, sondern vielmehr die genetische oder soziale Rolle, die mit der Beziehung einhergeht oder auf der die Beziehung basiert. Damit stellt sich jedoch die Frage, ob diese >formalen Eigenschaften< tatsächlich frei sind von jeglicher Normativität. Die Antwort, die ich an dieser Stelle vorschlage, lautet: Auch wenn in der genetischen Bindung oder der sozialen Bande selbst keine Normativität liegt, akzentuieren diese Bande respektive deren Eigenschaften die Verletzlichkeit, die Mitglieder in dieser sehr speziellen Beziehung einander gegenüber haben. Da Eltern und Kinder in einer gewissen Weise stets verbunden bleiben ganz unabhängig von ihren Vorlieben und Einstellungen, und da diese Beziehung unersetzlich ist, ist ein Scheitern dieser Bande schwieriger auszuhalten und ins Leben $\mathrm{zu}$ integrieren als ein Versanden einer Freundschaft.

\section{Verletzlichkeit und familiäre Pflichten}

Was heißt das nun für die Frage, was Kinder ihren Eltern schulden? Lässt sich aus der erwähnten Verletzlichkeit eine Verpflichtung ableiten und wenn ja welche?

Beginnen wir mit der epistemischen Verletzlichkeit. Es ist offensichtlich, dass Familienmitglieder einander in besonderer Weise verletzen können aufgrund des epistemischen Vorsprungs, den sie Außenstehenden gegenüber haben. Intimes Wissen voneinander verschafft Familienmitgliedern zwar auch die Möglichkeit, einander besonders zielführend zu helfen; doch dessen Kehrseite ist, dass sie auch in der Lage sind, einander zu verraten, zu beschämen, zu demütigen, wie es 
jemand, der diesen epistemischen Vorsprung nicht hat, nicht vermöchte. Es ist selbstredend verwerflich, die geteilte Intimität und das historische Wissen voneinander auszunutzen. Diese Pflicht ist jedoch eine natürliche Pflicht, die sich auf das generelle Nichtschädigungsgebot zurückführen lässt, wir haben diese Pflicht allen Personen gegenüber. Es handelt sich somit nicht um eine spezifisch filiale Pflicht, die Kinder ihren Eltern als ihre Kinder schulden. Die Intimität versetzt die Familienmitglieder lediglich in die Lage, sich besonders einfach und tiefgreifend verletzen zu können; sie ist aber nicht die Grundlage der besagten Pflicht.

Generiert die erwartungsbasierte Verletzlichkeit spezielle filiale Pflichten? Manche Freunde und auch Familienmitglieder teilen gemeinsame Projekte, in die beide Seiten Zeit und Geld investieren, wie etwa der Kauf einer gemeinsamen Ferienwohnung, die Planung einer Reise. Die Erwartungen, die auf diesen gemeinsamen Projekten basieren, fußen in einer Art implizitem Versprechen, das man sich gegeben hat. Schert einer der Projektpartner aus, schuldet er natürlich eine Rechtfertigung und muss den entstandenen Schaden kompensieren. Die Pflicht, sich an Versprechen zu halten und bei einem Vertragsbruch den Schaden zu kompensieren, ist aber wiederum eine generelle Pflicht; sie ist nicht familientypisch und kommt dort möglicherweise sogar seltener vor als in Freundschaften und vor allem in Geschäftsbeziehungen. Die Kosten, die für Familienmitglieder entstehen, wenn jemand einseitig den Kontakt abbricht oder jemand seinem Gegenüber nicht jene Fürsorge angedeihen lässt, die dieser sich wünscht, betreffen eher die emotionale Verletzlichkeit, die sich in Familienbeziehungen verschärft durch die Unaufkündbarkeit und Exklusivität. Doch folgt aus dieser, dass Kinder für ihre Eltern Verantwortung übernehmen müssen, dass sie zu Kontakt und Fürsorge verpflichtet sind?

Betrachten wir zur Beantwortung dieser Frage noch einmal die Freundschaft. Einander Gutes zu tun, einander zu ermutigen und gemeinsam Zeit zu verbringen, liegt Freunden normalerweise am Herzen. Weil Freunden an der Beziehung liegt, haben sie auch Grund, sich um diese zu bemühen. Doch dieser Grund ist keine Pflicht im engeren Sin- 
ne, die auch dann noch bestünde, wenn wir die Beziehung nicht mehr als wertvoll erachten. Ganz im Gegenteil: Würden wir uns nur noch aus einem Pflichtgefühl heraus um einander bemühen, hätten wir guten Grund uns zu fragen, ob wir tatsächlich noch Freunde sind. Das zeigt sich auch aus der drittpersonalen Perspektive: Wenn ich realisiere, dass meine Schwester ihre langjährige Freundin nicht an ihre Geburtstagsfeier eingeladen hat und nicht mehr mit ihr, wie früher immer, in die Ferien fährt, würde ich sie sicher nicht kritisieren für eine Vernachlässigung einer Pflicht. Vielmehr scheint es angebracht zu fragen, weshalb die Beiden nicht mehr befreundet sind. Eine Pflicht, befreundet zu sein und in einer Beziehung zu verharren, gibt es nicht, und dasselbe gilt auch für die Familienbeziehung.

Dieses Recht, eine Person zu verlassen, wird oft mit dem sogenannten Voluntarismus verteidigt: Es dürfen uns keine speziellen Pflichten aufgebürdet werden, ohne dass wir in die entsprechende Bande, die uns verpflichtet, eingewilligt hätten. Der Voluntarismus ist allerdings mit einer ganzen Reihe von Problemen konfrontiert, auf die ich an dieser Stelle nicht eingehen kann (zum Problem des Voluntarismus vgl. ausführlich: Scheffler 2002, Kap. 3). Das Konzept der Vulnerabilität kann jedoch meines Erachtens zeigen, weshalb es nicht nur erlaubt, sondern zuweilen sogar geboten ist, eine Person zu verlassen. Relationale Verletzlichkeit ist, so wie ich sie hier dargestellt habe, die Kehrseite jeder innigen Verbindung. Wenn die Liebe beidseits versiegt und die Bande nicht mehr als wertvoll erachtet wird, gibt es auch keinen Grund, diese weiter zu pflegen. Wenn die Liebe aber einseitig versiegt, dann sollte sich die Person, die gehen möchte, zwei Fragen stellen: Erstens eine kritische Rückversicherung: Ist die Beziehung wirklich ganz wertlos geworden? Gibt es tatsächlich keine Möglichkeit, sie weiterzuentwickeln, sondern bleibt nur der Abbruch, die Trennung? Immerhin gehören langanhaltende Beziehungen zum Wertvollsten im Leben, was es gibt. Wir haben deshalb prudentielle Gründe, auch kriselnden Verbindungen eine zweite und dritte Chance zu geben. Wenn die Beziehung aber wirklich wertlos geworden ist oder uns sogar belastet, dann haben wir genauso Grund, sie zu beenden. Wir müssen uns 
nicht nur nicht quälen in Beziehungen, wir sollten dies auch aus prudentiellen Gründen nicht tun. Doch auch moralische Gründe sprechen für einen Beziehungsabbruch, wenn wir nicht mehr in der Lage sind, ihr etwas Positives abzugewinnen. Wir haben festgehalten, dass relationale Verletzlichkeit die Kehrseite jeder liebevollen Verbindung ist. Ist die Liebe nicht mehr zu haben, wird die Verletzlichkeit, die vorher sozusagen der inbegriffene Preis für das hohe Gut der Liebe war, vollkommen nutzlos. Es wäre deshalb geradezu moralisch verwerflich, eine Person in einer Beziehung zu halten, in der wir ihr keine Liebe mehr geben können. Deshalb sollten wir nicht zuletzt aus Respekt vor der einst geliebten Person alles daransetzen, dass auch sie ihre emotionale Verletzlichkeit uns gegenüber überwinden kann.

Wenn wir nun wieder die Familienbeziehung betrachten, so ist es rein theoretisch natürlich möglich, dass Eltern wie auch Kinder ihr Interesse aneinander verlieren und sie beidseitig langsam aufhören, sich mit jenen Gefälligkeiten zu versorgen, die ihre Bindung immer wieder gefestigt hat. Daran ist nichts falsch - es ist bloß äußerst selten. In vielen Fällen erweist es sich aber als sehr schwierig, die relationale Verletzlichkeit in der Elternkindbeziehung zu überwinden. Der Grund dafür liegt in den speziellen Akzentuierungen, die die relationale Verletzlichkeit in dieser Beziehung erfährt: Nichtaufkündbarkeit und Exklusivität. Man kann nie ganz über diese Beziehung >hinwegkommen<; insofern ist Blut tatsächlich dicker als Wasser.

In Familien ist die Verletzlichkeit auch gar nicht so sehr die Kehrseite der Liebe (oder nicht ausschließlich), sondern die Kehrseite einer sehr viel breiteren Form der Zugehörigkeit, die Identität, Biographie, eine ganze Reihe von weiteren Verwandten sowie soziale Konventionen und öffentliche Anerkennung umfassen. Einander aufzugeben oder $\mathrm{zu}$ verlieren, ist in Familien deshalb besonders gravierend, denn man verliert mit ihr einen ganzen Teil der eigenen Geschichte und Identität. Kinder haben deshalb sicherlich Grund, sich um ihre Eltern zu kümmern - einen Grund, der nicht allein in der Wertschätzung der Beziehung liegt, wie es bei der Freundschaft der Fall ist, sondern darüber hinaus einen Grund, der mit ihrer spezifischen Vulnerabilität zu tun hat. 
Vielleicht scheint dieses Bild, das ich hier von der Familienbande zeichne, zu düster. Aber wie gesagt, stellen sich moralische Fragen in Beziehungen meist erst im Falle deren Störung, angesichts von Spannungen, Missverständnissen, Vertrauensbrüchen. Dies gilt nicht nur für Liebesbeziehungen und Freundschaften, sondern auch in der Familie. Die Spannungen und Verletzungen, die Familiengeschichten mit sich führen, thematisieren wir ungern. Aber eine Romantisierung der Familienbande übersieht, dass gerade in diesen Beziehungen die schlimmsten Dinge passieren können - denken wir an Missbrauch, Gewalt in der Ehe, erweiterte Suizide in Familien. Es ist deshalb vonnöten, diese Seite der Familiengeschichten ebenfalls in den Blick zu nehmen und in die Frage nach filialer Verantwortung einzubeziehen. Wie ich zu zeigen versucht habe, meine ich, dass der Begriff der Verletzlichkeit für dieses Vorhaben hilfreich und zielführend sein kann.

Der hier gemachte Vorschlag, wie wir familiäre Verantwortung denken sollten, macht einen weiteren wichtigen Punkt deutlich, nämlich, dass die Verletzlichkeit und die entsprechenden Pflichten auf beiden Seiten - auf der Seite der Kinder wie auf der Seite der Eltern - bestehen. Anders als das Reziprozitäts- und das Dankbarkeitsmodell, die nur die erwachsenen Kinder in die Pflicht nehmen, verlangt das Verletzlichkeitsmodell von Allen an der Beziehung Beteiligten einen sorgsamen Umgang miteinander. Man muss nur einmal Franz Kafkas Brief an seinen Vater lesen, um zu verstehen, weshalb diese Anmahnung ihre bittere Berechtigung hat (vgl. Kafka 1991: 5).

Die Rede von relationaler Vulnerabilität zeigt aber auch, dass die Frage nach der filialen Verantwortung nicht adäquat beantwortet werden kann, wenn wir nur danach fragen, was Kinder ihren Eltern schulden, sondern dass stets auch die erwachsenen Kinder selbst sich fragen müssen, was sie in ihrer jeweiligen Verletzlichkeit leisten können und wo ihre jeweiligen Grenzen verlaufen. Denken wir zurück an die Rede vom Riskanten, das jeder Beziehung anhaftet: Beziehungen können nicht nur scheitern, sie können auch ausbeuten, demütigen und verletzen. Dann haben erwachsene Kinder ihrerseits selbstverständlich allen Grund, Distanz zu wahren und sich selber zu schützen. Insofern sich 
Eltern und Kinder aber nie ganz lassen können, weil sie stets aneinander Gebundene bleiben, spricht viel dafür, lange an der Beziehung zu arbeiten und die Verantwortung für diese unter Beachtung der gegenseitigen Vulnerabilität wahrzunehmen. ${ }^{3}$

3 Ich danke Prof. Mathias Lindenau und dem Zentrum für Ethik \& Nachhaltigkeit ZEN-FHS für die ehrenvolle Einladung, eine der Vadian Lectures halten zu dürfen. Danke auch dem Publikum für die klugen Fragen und die rege Diskussion nach dem Vortrag. 


\section{LITERATUR}

Archard, David (2015): »Das Ende der Familie? Zur Bedeutung der biologischen Verwandtschaft«, in: Betzler/Bleisch, Familiäre Pflichten, S. 57-86.

Aristoteles (1995): Die Nikomachische Ethik, Neuntes Buch, Kap. 1-2, übersetzt u. eingeleitet v. Olof Gigon, 2. Aufl., München, S. 307311.

Bernhard, Andreas (2014): Kinder machen. Neue Reproduktionstechnologien und die Ordnung der Familie, Frankfurt a.M.

Betzler, Monika/Bleisch, Barbara (Hg.) (2015): Familiäre Pflichten, Frankfurt a.M.

Betzler, Monika/Bleisch, Barbara (2015): »Einleitung«, in: Dies. (Hg.), Familiäre Pflichten, S. 9-54.

Bleisch, Barbara (2015): »Rabentöchter? Rabensöhne? Zur Begründung filialer Pflichten«, in: Zeitschrift für praktische Philosophie 2(2), S. 237-272.

Butler, Judith (2004): Precarious life. The powers of mourning and violence, London.

Gheaus, Anca (2015): »Hat die Familie einen besonderen Wert?«, in: Betzler/Bleisch, Familiäre Pflichten, S. 87-104.

Haslanger, Sally (2009): »Family, Ancestry and Self. What is the Moral Significance of Biological Ties?«, in: Adoption \& Culture 2, S. 92-122.

Kafka, Franz (1991): Brief an den Vater, Frankfurt a.M.

Mackenzie, Catriona/Stoljar, Natalie (2000): »Introduction: Autonomy Refigured «, in: Dies. (Eds.), Relational Autonomy. Feminist Perspective on Autonomy, Agency, and the Social Self, New York, S. 3-31.

MacIntyre, Alasdair (1999): Dependent rational animals. Why human beings need the virtues, Chicago.

Mills, Claudia (2015): »Pflichten gegenüber älter werdenden Menschen «, in: Betzler/Bleisch, Familiäre Pflichten, S. 260-281. 
Montaigne, Michel de (1998): »Über die Freundschaft«, in: Ders., Essais, hrsg. v. Hans Magnus Enzensberger, Frankfurt a.M., S. 98104.

Scheffler, Samuel (2002): Boundaries and Allegiances. Problems of Justice and Responsibility in Liberal Thought, Oxford.

Velleman, David (2005): »Family History«, in: Philosophical Papers 34 (3), S. 357-378. 
